\title{
Quantum Chinos Game: winning strategies through quantum fluctuations
}

\author{
F. Guinea ${ }^{\dagger}$ and M.A. Martín-Delgado ${ }^{\ddagger}$ \\ ${ }^{\dagger}$ Instituto de Ciencia de Materiales de Madrid, CSIC, Cantoblanco, E-28049 Madrid, Spain. \\ ${ }^{\ddagger}$ Departamento de Física Teórica I, Universidad Complutense, 28040. Madrid, Spain.
}

\begin{abstract}
We apply several quantization schemes to simple versions of the Chinos game. Classically, for two players with one coin each, there is a symmetric stable strategy that allows each player to win half of the times on average. A partial quantization of the game (semiclassical) allows us to find a winning strategy for the second player, but it is unstable w.r.t. the classical strategy. However, in a fully quantum version of the game we find a winning strategy for the first player that is optimal: the symmetric classical situation is broken at the quantum level.
\end{abstract}

PACS numbers: 03.67.-a, 03.67.Lx

In a typical scene at a Spanish restaurant, a small group of companions-at-table gather at the bar extending their arms, each with their clenched hands holding a few coins hidden inside. They are gambling for the after-lunch round of coffees. One after another they tell a number, then open their hands showing their coins one another and count them all. Ofently, one of the pals smile meaning that $\mathrm{s} /$ he guessed the correct total number of coins. After a given number of plays, the player scoring the worst pays for all coffees. This gambling game is known as the Chinos game and has been a traditional way in Spain to decide who is in charge for the coffees' check [1].

Interestingly enough, this simple-minded guessing game exhibits a rich variety of patterns with complex behaviour that has been used to model strategic behaviour in some social and economic problems, like financial markets and information transmission [2]. This is an example of non-cooperative game for each player seeks to maximize her/his chances of guessing correctly, and at the same time to minimize the possibilities of her/his opponents.

Recently, a new field for game theory has emerged in the form of quantum games with the goal of taking advantage of quantum effects to attain a winning edge [3], [1], [5]. The blending of quantum mechanics with game theory opens novel strategies based in exploiting the peculiarities of quantum behaviour, and it has already estimulated a number of new ideas, e.g., in the Prisioners' Dilemma there exists a quantum strategy that allows both players to escape the dilemma何.

In this letter our aim is twofold: firstly, to define quantum versions of the Chinos game such that they reduce to the classical game as a limiting case. Secondly, to analyse the new quantum versions in order to find how the classical strategies behave under quantum effects, and if there exists new quantum winning strategies without classical analogue.
Classical Chinos Game. In the classical formulation, a number $N_{\mathrm{p}}$ of players enter the game, each having access to $N_{\mathrm{c}}$ coins that they draw and hide in their hands at each round of the game. Next, each player makes a guess about the total number of coins held at that round, with the constraint that no player can repeat the number guessed by the previous players. Thus, the outcome of a given round may be either that one player wins or failure for everyone. As a remark, the heads and tails of the coins play no role in the Chinos game, so that they can be simply regarded as pebbles: only their number count.

Let $\mathcal{D}:=\left\{0,1, \ldots, N_{\mathrm{c}}\right\}$ be the space of draws and $\mathcal{G}:=\left\{0,1, \ldots, N_{\mathrm{p}} N_{\mathrm{c}}\right\}$ be the space of guesses for the first player. Each players' movement has two parts: 1/ drawing coins; 2/ guessing the total number of coins altogether. Let us denote by $M:=(d, g)$ one of these movements, with $d \in \mathcal{D}$ and $g \in \mathcal{G}$. The space of movements is $\mathcal{M}:=\mathcal{D} \times \mathcal{G}$ for the first player. Next players have a reduced guess space $\mathcal{G}_{(i)}^{\prime}:=\mathcal{G}-\left\{d_{(1)}, \ldots, d_{(i-1)}\right\}, i=$ $2, \ldots, N_{\mathrm{p}}$. A possible strategy $S$ is an ordered sequence of movements $S:=\left(M_{1}, M_{2}, \ldots, M_{r}\right)$ selected with some criteria or randomly, and played during the $r$ rounds that the whole game takes.

We shall denote by $\operatorname{CCG}\left(N_{\mathrm{p}}, N_{\mathrm{c}}\right)$ a classical Chinos game of $N_{\mathrm{p}}$ players and $N_{\mathrm{c}}$ coins. The exhaustive analysis of such a generic game turns out to be too complicated [2], thus we shall concentrate on the case of only $N_{\mathrm{p}}=2$ players for which we have the following result:

$1^{\text {st }}$ Result. Let us denote the classical strategies for each player $i=1,2$ by $S_{(i)}:=\left(M_{(i), 1}, M_{(i), 2}, \ldots, M_{(i), r}\right)$. Then, the best strategy for player 1 is to choose movements $M_{(1), j}, j:=1,2, \ldots, r$ with $d_{(1), j}$ randomly distributed and $g_{(1), j}=N_{\mathrm{c}}, \forall j$, while the best strategy for player 2 is to choose draws $d_{(2), j}$ at random. For $r$ large enough, the result of the game is even.

Proof. Since the Chinos game is a non-cooperative game, in this result we are assuming that one of the main goals of player 1 is not to transmit any informa- 
tion to player 2 about her $/$ his values $d_{(1), j}$. This can be achieved by choosing $g_{(1), j}=N_{\mathrm{c}}$ irrespective of the number that $\mathrm{s} /$ he draws. Moreover, players soon realize that as they cannot know in advance her/his opponent strategy, the best strategy they can choose is to pick $d_{(i), j}, i=1,2 ; \forall j$ at random. Now, let us call $p_{1}$ the probability that player 1 guesses correctly the total sum they are after, namely, $a_{j}:=d_{(1), j}+d_{(2), j}$, and similarly for $p_{2}$. The quantities each player is interested in maximizing are the normalized probabilities $P_{i}:=p_{i} / \sum_{i=1,2} p_{i}$. Thus, under these circumstances, the probability that the second player guesses the correct sum is

$$
p_{2}=\frac{1-p_{1}}{N_{\mathrm{c}}} .
$$

Then, the quantity player 2 wants to optimize is

$$
P_{2}=\frac{1-p_{1}}{1+p_{1}\left(N_{\mathrm{c}}-1\right)},
$$

which is a decreasing function of $p_{1}$, so that player 2 is interested in reducing $p_{1}$ as much as possible. However, player 1 can always resort to make random guesses about the number of coins drawn by player 2 . This amounts to a lowest bound on $p_{1}$ given by $p_{1,<}:=1 /\left(N_{\mathrm{c}}+1\right)$. Therefore, player 2 should draw coins at random so that $p_{1}$ cannot exceed $p_{1,<}$ and we end up with an even situation given by [6]

$$
P_{1}=P_{2}=\frac{1}{2}
$$

We may view this result as a sort of "classical symmetry" between players 1 and 2 :

$$
\text { Player } 1 \longleftrightarrow \text { Player } 2,
$$

in the sense that there is now way to untight the result of the game if both players play at random. Our goal is to define quantum extensions of the Chinos game to see if this symmetry can be broken at the quantum level. We shall use this classical result as a guide to analyse the behaviour of classical strategies when we enter the realm of the quantum extensions of the game.

Semiclassical Chinos Game. A first attempt at quantizing the Chinos game is to make a quantum extension of the space of draws $\mathcal{D}_{q}$ while leaving the space of guesses $\mathcal{G}$ classical. We term this case semiclassical for obvious reasons and denote by $\operatorname{SCG}\left(N_{\mathrm{p}}, N_{\mathrm{c}}\right)$ a semiclassical Chinos game. The natural choice for $\mathcal{D}_{q}$ is to replace coins by quantum coins or qubits. Likewise, a quantum two-level system is represented by a spin $\frac{1}{2}$ particle with states $|\uparrow\rangle,|\downarrow\rangle$ representing heads and tails, respectively. However, we find that spins are not appropriate in the Chinos game since only the presence or absence of coins in players' hands matters. Hence, a more suitable way of representing qubits is to use a boson system defined by bosonic creation/annihilation operators $b^{\dagger}, b$ obeying canonical commutation relations (CCR) $\left[b, b^{\dagger}\right]=1$ and acting on the bosonic vacuum $|0\rangle$ in the standard fashion: $b|0\rangle=0, b^{\dagger}|n\rangle=\sqrt{n+1}|n+1\rangle$, with $|n\rangle:=\left(b^{\dagger}\right)^{n}|0\rangle / \sqrt{n !}$.

For simplicity, we shall consider first the case in which each quantum player has only one coin, namely, $\operatorname{SCG}\left(N_{\mathrm{p}}, 1\right)$.

To each player $i=1,2, \ldots, N_{\mathrm{p}}$ we shall assign a set of operators $O\left(\theta_{i}, \phi_{i}\right)$ parameterized by the two angles characterizing a qubit state in the Bloch sphere. Thus, we introduce

$$
O_{i}(\theta, \phi):=\cos \frac{1}{2} \theta_{i}+\mathrm{e}^{\mathrm{i} \phi_{i}} \sin \frac{1}{2} \theta_{i} b^{\dagger}, 2 \theta_{i}, \phi_{i} \in[0,2 \pi) .
$$

These operators represent the quantum draw space $\mathcal{D}_{q}$. At a given round $j$ of the game, each player selects one possible operator $O_{i}(\theta, \phi)$ and at the end of the drawing process, we represent the situation of having all players' hands together by the following joint quantum state

$$
\left|\Psi_{\mathrm{SCG}}^{\left(N_{\mathrm{p}}, 1\right)}\right\rangle:=N^{-1 / 2} \prod_{i=1}^{N_{\mathrm{p}}} O_{i}(\theta, \phi)|0\rangle=\sum_{n=0}^{N_{\mathrm{p}}} c_{n}|n\rangle,
$$

where $N$ is a normalization constant and $c_{n}$ expansion coefficients. This state faithfully represents the fact that what really counts is to guess the total sum $a_{j}=\sum_{i=1}^{N_{\mathrm{p}}} d_{(i), j} \in \mathcal{G}$, no matter what the partial contributions $d_{(i), j}$ of each player are. Moreover, the quantum effects are clearly apparent since when the state $\left|\Psi_{\mathrm{SCG}}^{\left(N_{\mathrm{p}}, 1\right)}\right\rangle$ is expanded in states $|n\rangle, n \in \mathcal{G}$, each coefficient $c_{n}$ receives contributions from each player that cannot be factorized out. Then, with (6) we can compute the probability $p(n)$ that any player obtains the value $g=n$ after a measurement, namely,

$$
p(n):=\left|\left\langle n \mid \Psi_{\mathrm{SCG}}^{\left(N_{\mathrm{p}}, 1\right)}\right\rangle\right|^{2}=c_{n}^{2} n !
$$

With the present quantization scheme we have an infinitely many number of possible draws. In practice, it is a reasonable assumption to reduce the possible operator choices to a finite restricted set. To be concrete, let us consider the case of $N_{\mathrm{p}}=2$ players $\operatorname{SCG}(2,1)$ and we select from (6) the following reduced operator set

$$
\begin{aligned}
& O_{1}:=I, O_{2}:=\frac{1}{\sqrt{2}}\left(I+b^{\dagger}\right), \\
& O_{3}:=\frac{1}{\sqrt{2}}\left(I-b^{\dagger}\right), O_{4}:=b^{\dagger} .
\end{aligned}
$$

Notice that operators $O_{1}$ and $O_{4}$ represent the classical draws of 0 and 1 , respectively, while $O_{2}$ and $O_{3}$ represent novel quantum superpositions of the classical draws. These conditions represent a generic situation to analyse quantum effects in the Chinos game and we find the following result: 


\begin{tabular}{||c||c|c|c|c||}
\hline \hline & $O_{1}^{(1)}$ & $O_{2}^{(1)}$ & $O_{3}^{(1)}$ & $O_{4}^{(1)}$ \\
\hline \hline \multirow{3}{*}{$O_{1}^{(2)}$} & $p(0)=1$ & $p(0)=\frac{1}{2}$ & $p(0)=\frac{1}{2}$ & $p(0)=0$ \\
& $p(1)=0$ & $p(1)=\frac{1}{2}$ & $p(1)=\frac{1}{2}$ & $p(1)=1$ \\
& $p(2)=0$ & $p(2)=0$ & $p(2)=0$ & $p(2)=0$ \\
\hline \multirow{3}{*}{$O_{2}^{(2)}$} & $p(0)=\frac{1}{2}$ & $p(0)=\frac{1}{7}$ & $p(0)=\frac{1}{3}$ & $p(0)=0$ \\
& $p(1)=\frac{1}{2}$ & $p(1)=\frac{4}{7}$ & $p(1)=0$ & $p(1)=\frac{1}{3}$ \\
& $p(2)=0$ & $p(2)=\frac{2}{7}$ & $p(2)=\frac{2}{3}$ & $p(2)=\frac{2}{3}$ \\
\hline \multirow{3}{*}{$O_{3}^{(2)}$} & $p(0)=\frac{1}{2}$ & $p(0)=\frac{1}{3}$ & $p(0)=\frac{1}{7}$ & $p(0)=0$ \\
& $p(1)=\frac{1}{2}$ & $p(1)=0$ & $p(1)=\frac{4}{7}$ & $p(1)=\frac{1}{3}$ \\
& $p(2)=0$ & $p(2)=\frac{2}{3}$ & $p(2)=\frac{2}{7}$ & $p(2)=\frac{2}{3}$ \\
\hline \multirow{2}{*}{$O_{4}^{(2)}$} & $p(0)=0$ & $p(0)=0$ & $p(0)=0$ & $p(0)=0$ \\
& $p(1)=1$ & $p(1)=\frac{1}{3}$ & $p(1)=\frac{1}{3}$ & $p(1)=0$ \\
& $p(2)=0$ & $p(2)=\frac{2}{3}$ & $p(2)=\frac{2}{3}$ & $p(2)=1$ \\
\hline \hline
\end{tabular}

TABLE I: Probabilities for the outcomes of total coins 0,1 and 2 in a $\operatorname{SCG}(2,1)$ game. In the horizontal, the draws of player 1 and in the vertical, the draws for player 2 .

$2^{\text {nd }}$ Result. i) The strategy of drawing randomly from (8) becomes a winning strategy for player 2. However, this strategy is unstable. ii) The classical strategy of drawing randomly between $O_{1}$ and $O_{4}$ is a winning strategy for both players (evenness) and is stable.

Proof. The analysis relies on Table 1 showing the probabilities of obtaining 0,1 and 2 coins when player 1 draws operator $O_{i_{1}}^{(1)}$ and player 2 draws $O_{i_{2}}^{(2)}, i_{1}, i_{2}=1,2,3,4$, according to (6)-(8). i/ Let us assume that players 1 and 2 both know the classical winning strategy of a CCG and decide to make a straightforward generalization of it to the semiclassical case SCG. Then, player 2 decides to play random draws among the four possible choices in (8). In this situation, player 1 is left with a set of probabilities of getting a number of coins 0,1 and 2 given by Table III, which are computed from Table If by tracing out (averaging) over player 2. Hence, if the second player plays at random, the best choice for player 1 is to guess 1 (or $0)$ if s/he draws $O_{1}^{(1)}$, and 2 if s/he draws $O_{2}^{(1)}, O_{3}^{(1)}$ and $O_{4}^{(1)}$. Thus, her/his total chances of winning are:

$$
P_{1}=\frac{1}{4} \times \frac{1}{2}+\frac{1}{2} \times \frac{68}{168}+\frac{1}{4} \times \frac{7}{12}=\frac{53}{112}<\frac{1}{2}
$$

Therefore, the strategy of both players drawing at random is no longer an even strategy in this case.

ii/ However, after a large number of rounds $r$, player 1 will realize that playing at random is a winning strategy for her/his opponent and then s/he will seek to improve it. To do this, s/he may resort to draw only the classical

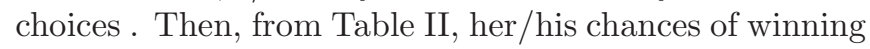
are

$$
P_{1}=\frac{1}{2} \times \frac{1}{2}+\frac{1}{2} \times \frac{7}{12}=\frac{13}{24}>\frac{1}{2}
$$

This implies that the strategy in i) is not stable. Likewise, player 2 will not be happy with this new situation. S/he

\begin{tabular}{||c|c|c|c||}
\hline \hline$O_{1}^{(1)}$ & $O_{2}^{(1)}$ & $O_{3}^{(1)}$ & $O_{4}^{(1)}$ \\
\hline \hline$\langle p(0)\rangle=\frac{1}{2}$ & $\langle p(0)\rangle=\frac{41}{168}$ & $\langle p(0)\rangle=\frac{41}{168}$ & $\langle p(0)\rangle=0$ \\
$\langle p(1)\rangle=\frac{1}{2}$ & $\langle p(1)\rangle=\frac{59}{168}$ & $\langle p(1)\rangle=\frac{59}{168}$ & $\langle p(1)\rangle=\frac{5}{12}$ \\
$\langle p(2)\rangle=0$ & $\langle p(2)\rangle=\frac{68}{168}$ & $\langle p(2)\rangle=\frac{68}{168}$ & $\langle p(2)\rangle=\frac{7}{12}$ \\
\hline \hline
\end{tabular}

TABLE II: Averaged probabilities of obtaining 0,1, and 2 coins by player 1 in a $\operatorname{SCG}(2,1)$ game according to the draws $O_{i}, i=1,2,3,4 \mathrm{~s} /$ he makes.

will try to match player's 1 strategy by choosing the same purely classical strategy. This fully classical situation is represented by the boxes at the outer corners of Table if. Then we are led to $P_{1}=P_{2}=\frac{1}{2}$ as the stable best strategy as in (3).

This result means that if player 1 applys her/his knowledge of the classical game naively by drawing at random from the four choices available, in the long run s/he will realize that player 2 gets a winning edge.

Quantum Chinos Game. Motivated by the previous semiclassical analysis, we propose a fully quantized version of the Chinos game by quantizing both the draw space $\mathcal{D}_{q}$ and the guessing space $\mathcal{G}_{q}$. We shall define the quantum space of guesses $\mathcal{G}_{q}$ by allowing each player to make a guess about the final quantum state $\left|\Psi_{\mathrm{SCG}}^{\left(N_{\mathrm{p}}, 1\right)}\right\rangle$ in (6), and not merely about the possible outcomes of the total coins. Thus, each player $i$ will make a guess $\left|\Psi_{i}\right\rangle, i=1,2, \ldots, N_{\mathrm{p}}$ about what the actual joint quantum state they are dealing with. Moreover, we also extend quantumly the classical constraint that the guess $g_{i}$ of player $i$ cannot be the same as guesses $g_{j}$ for $i<j$. This is achieved by imposing that the guess a player $i$ can make is restricted to the subspace orthogonal to the space spanned by the guesses of the previous players, namely,

$$
\mathcal{G}_{q, i}:=\operatorname{span}\left\{\left|\Psi_{1}\right\rangle, \ldots,\left|\Psi_{i-1}\right\rangle\right\}^{\perp} .
$$

With these new rules, we need to define a new function payoff: the gain for player $i$ is

$$
f_{i}:=\left|\left\langle\Psi_{i} \mid \Psi_{\mathrm{SCG}}^{\left(N_{\mathrm{p}}, 1\right)}\right\rangle\right|^{2} .
$$

This way of quantizing the space of guesses is reminiscent of the theory of quantum algorithms [7, and more specifically, from quantum searching algorithms [8], [9]. That this fully quantum version of the Chinos game includes the classical one is guaranteed since the latter appears as a particular case when the only allowed guesses are $|0\rangle,|1\rangle, \ldots,\left|N_{\mathrm{p}}\right\rangle$ (if the number of coins per player is $N_{\mathrm{c}}=1$.)

For simplicity, we shall consider the quantum case QCG $\left(N_{\mathrm{p}}, N_{\mathrm{c}}\right)$ for two players and one coin each, and their quantum guesses comprise the finite set (8). We find the following result: 
$3^{\text {rd }}$ Result. In a quantum Chinos game $\operatorname{CCG}(2,1)$, the first player has a stable winning strategy that allows her/his to win more than half of the times.

Proof. A systematic analysis in this case of 2 players with one coin each proceeds as follows. The space of draws for player 1 is $\mathcal{D}_{q}$ given by the reduced set ( 8 ). Then, player 1 draws $O_{i_{1}}^{(1)} \in \mathcal{D}_{q}$. The space of guesses for player 1 is $\mathcal{G}_{q, 1}:=\left\{O_{j_{1}}^{(1)} O_{k_{1}}^{(1)}, 1 \leq j_{1} \leq k_{1} \leq 4\right\}$. Next, player 1 makes a quantum guess $g_{q, 1}:=O_{j_{1}}^{(1)} O_{k_{1}}^{(1)} \in \mathcal{G}_{q, 1}$. Now enters player 2 with a draw $O_{i_{2}}^{(2)} \in \mathcal{D}_{q}$, and making a guess $g_{q, 2}:=O_{j_{2}}^{(2)} O_{k_{2}}^{(2)}$ that in order to be eligligible, it has to be orthogonal to player's 1 guess (11). To characterize this orthogonality condition, it is convenient to introduce the following $16 \times 16$ matrix

$$
\begin{aligned}
G_{\left(j_{1}, k_{1}\right),\left(j_{2}, k_{2}\right)} & :=\frac{\left\langle 0\left|O_{j_{1}}^{\dagger} O_{k_{1}}^{\dagger} O_{j_{2}} O_{k_{2}}\right| 0\right\rangle}{\sqrt{N_{j_{1} k_{1}}} \sqrt{N_{j_{2} k_{2}}}}, \\
N_{j k} & :=\left\langle 0\left|O_{j}^{\dagger} O_{k}\right| 0\right\rangle,
\end{aligned}
$$

which can be thought of as a metric on the quantum guess space. Thus, guess $g_{q, 2}$ is admissible for the given guess $g_{q, 1}$ iff $G_{\left(j_{1}, k_{1}\right),\left(j_{2}, k_{2}\right)}=0$. Finally, for a pair of draws, the actual joint state representing that round of the game is

$$
\left|\Psi_{\mathrm{QCG}}^{(2,1)}\right\rangle=N_{12}^{-1 / 2} O_{i_{1}}^{(1)} O_{i_{2}}^{(2)}|0\rangle,
$$

while the function payoffs $f_{i}, i=1,2$ for each player can also be read off from the metric (13) as follows

$$
\begin{aligned}
& f_{1}=\left|G_{\left(j_{1}, k_{1}\right),\left(i_{1}, i_{2}\right)}\right|^{2}, \\
& f_{2}=\left|G_{\left(j_{2}, k_{2}\right),\left(i_{1}, i_{2}\right)}\right|^{2} .
\end{aligned}
$$

Then, once we have computed the metric (13), it is possible to make an exhaustive study of all the possibilities in this quantum Chinos gain and compute each players' payoffs for each of those possibilities. We have performed this analysis with the following result: let us show that if player 1 makes draws with equal probability among the choices $O_{2}^{(1)}$ and $O_{3}^{(1)}$ only (8), then s/he is half-way for a winning position. The rest of the strategy is to set up the quantum guesses as follows. When player 1 draws $\mathrm{O}_{2}^{(1)}, \mathrm{s} /$ he decides to make always the following quantum guess

$$
\left|\Psi_{1}\right\rangle:=N_{1}^{-1 / 2} O_{2}^{(1)} O_{2}^{(1)}|0\rangle=\frac{1}{\sqrt{7}}(|0\rangle+2|1\rangle+\sqrt{2}|2\rangle),
$$

in which case, a possible guess for player 2 according to (11) would be

$$
\left|\Psi_{2}\right\rangle:=N_{2}^{-1 / 2} O_{3}^{(2)} O_{4}^{(2)}|0\rangle=\frac{1}{\sqrt{3}}(|1\rangle-\sqrt{2}|2\rangle) .
$$

While if s/he draws $O_{3}^{(1)}$, s/he decides to make always the following quantum guess

$$
\left|\Psi_{1}\right\rangle:=N_{1}^{-1 / 2} O_{3}^{(1)} O_{3}^{(1)}|0\rangle=\frac{1}{\sqrt{7}}(|0\rangle-2|1\rangle+\sqrt{2}|2\rangle) .
$$

Now, let us analyse the case when player 1 draws $O_{2}^{(1)}$. Then, player 2 is left with the four draws in the set (8) and the correspoding joint final states $\left|\Psi_{\text {SCG }}^{(2,1)}\right\rangle$ that we collet in Table III. When the first player draws $O_{3}^{(1)}$, then we obtain a similar table by exchanging $2 \leftrightarrow 3$.

¿From Table III we see that under these circumstances, it is clear that player 2 will avoid to make the classical draws $O_{1}^{(2)}$ and $O_{4}^{(2)}$, since they yield payoffs $f_{1}=\frac{9}{14}>$ $\frac{1}{2}, f_{1}=\frac{16}{21}>\frac{1}{2}$ for the first player. Thus, player 2 is led to play only the draws $O_{2}^{(2)}$ and $O_{3}^{(2)}$ at random. However, even in this case, player 1 will have a winning edge on the average since the chances of winning for the first player are

$$
\left\langle f_{1}\right\rangle=\frac{1}{2} \times 1+\frac{1}{2} \times \frac{1}{21}=\frac{11}{21}>\frac{1}{2} .
$$

\begin{tabular}{||c|c|c||}
\hline \hline Quantum guess & Joint state $\left|\Psi_{\mathrm{CCG}}^{(2,1)}\right\rangle$ & Gain for player 1 \\
\hline \hline$O_{1}^{(2)}$ & $\frac{1}{\sqrt{2}}(|0\rangle+|1\rangle)$ & $f_{1}=\frac{9}{14}$ \\
\hline$O_{2}^{(2)}$ & $\frac{1}{\sqrt{7}}(|0\rangle+2|1\rangle+\sqrt{2}|2\rangle)$ & $f_{1}=1$ \\
\hline$O_{3}^{(2)}$ & $\frac{1}{\sqrt{3}}(|0\rangle-\sqrt{2}|2\rangle)$ & $f_{1}=\frac{1}{21}$ \\
\hline$O_{4}^{(2)}$ & $\frac{1}{\sqrt{3}}(|1\rangle+\sqrt{2}|2\rangle)$ & $f_{1}=\frac{16}{21}$ \\
\hline \hline
\end{tabular}

TABLE III: Quantum guesses for player 2 when player 1 draws $O_{2}^{(1)}(8)$, and the corresponding joint state (14) and gains for player 1 (15).

Conclusions. In game theory, players strive for even the slightest advantage that would tilt a game's outcome in their favor. We have found that the chances of winning for player 1 are better off on average than those of her/his opponent. We may interpret this result as the breaking of the symmetric classical situation (4) at the quantum level:

$$
\text { Player } 1 \longleftrightarrow \text { Player } 2 \text {. }
$$

This advantage of the first player resembles a similar situation found in the PQ quantum game 3]. In the present case, however, the correlation between players in the final result is dynamically generated, i.e., it is a consequence of the player's choice, and it is not encoded in the initial state. In this respect, it also differs from the quantum generalization of other simple games, like the prisoner's dilemma 4 , or the minority game 10 .

Acknowledgments. We acknowledge financial support from projects: PGC96-0875, 07N/0015/2001 and PB980685 . 
[1] We do not claim that the Chinos game originates from Spain, but the word chinos is a deformation of chinas meaning pebbles in Spanish.

[2] L. Pastor-Abia, M Pérez-Jordá, E. San-Fabián, E. Louis, F. Vega-Redondo, Adv. Complex Systems 1, 1 (2000).

[3] D. Meyer, Phys. Rev. Lett. 82, 1052 (1999).

[4] J. Eisert, M. Wilkens, M. Lewenstein, Phys. Rev. Lett. 83, 3077 (1999).

[5] For a review on quantum games, see E. Klarreich, Nature 414, 244 (2001).

[6] If player 1 gives away some information about the coins $\mathrm{s} /$ he draws to player 2 , then $p_{2}>\frac{1-p_{1}}{N}$ and $P_{1}<\frac{N_{c} p_{1}}{1-p_{1}\left(N_{c}-1\right)}$. As player 1 wants $P_{1}>\frac{1}{2}$, then it follows that $p_{1}>\frac{1}{N_{\mathrm{c}}+1}$. On the contrary, player 2 wants to minimize $P_{1}$ by drawing coins at random so that $p_{1}=\frac{1}{N_{\mathrm{c}}+1}$, bringing the situation back to the case (3).

[7] For a review on quantum algorithms, see A. Galindo, M.A. Martín-Delgado, quant-ph/0112105; Rev. Mod. Phys. to appear.

[8] L. K. Grover, Phys. Rev. Lett. 78, 325 (1997).

[9] D. Meyer, AMS Contemporary Mathematics volume: Quantum Computation and Quantum Information Science; quant-ph/0004092.

[10] R. Kay, N.F. Johnson, and S.C. Benjamin, quant$\mathrm{ph} / 0102008$. 